\title{
Estudio mediante SEM/EDS del taladrado en seco de la aleación Ti-6Al-4V
}

\author{
J.L. Cantero*, J.M. Sánchez-Sola*, M.M. Tardío*, M.A. Sebastián***, M.S. Carrilero*, \\ M. Marcos* y M.H. Miguélez
}

Resumen

En este artículo se estudia el proceso de taladrado sin fluido de corte, de la aleación Ti6Al4V, comparando dos condiciones de mecanizado, con y sin enfriamiento de herramienta y material entre taladros. El objetivo de este trabajo es analizar la influencia en el desgaste de la herramienta y en la calidad del agujero, del diferente incremento de temperatura originado por trabajar en una u otra condición. Para ello, se realizará análisis SEM/EDS y microscopía óptica de la broca e inspección visual del material.

Palabras clave Taladrado. Titanio. Mecanizado en seco. SEM/EDS. Desgaste de herramienta.

\section{Tool wear in the dry drilling of Ti-6Al-4V. A SEM/EDS study}

\begin{abstract}
This paper is focused on dry drilling of Ti6Al4V. In absence of cutting fluid, high temperatures are reached. Two different cutting conditions are studied in order to analyse the influence of temperature. In first condition, tests with no pause between drilled holes were carried out. In second condition, tests were performed cooling the tool with air between consecutively holes. Tool wear was studied with optical microscope and SEM-EDS techniques. The quality of machined holes was estimated in terms of optical inspection. Significantly differences in tool wear evolution were observed between both cutting conditions analysed.
\end{abstract}

Keywords Drilling. Titanium. Dry machining. SEM/EDS. Tool wear.

\section{INTRODUCCIÓN}

El mecanizado en seco o con tecnologías ecológicas de refrigeración y lubricación ha despertado interés en diversos grupos de trabajo en los últimos años ${ }^{[1-3]}$. El uso del fluido de corte produce contaminación y representa un porcentaje significativo del coste del mecanizado, por lo que su eliminación, reducción o sustitución por un sistema de refrigeración mejorado, siempre es deseable $^{[4]}$. Sin embargo, la ausencia o reducción del fluido de corte, que se traduce en falta de lubricación y refrigeración, condiciona los parámetros de corte y el tipo de herramientas a utilizar, que han de adaptarse a las nuevas condiciones, muy agresivas para la herramienta y para el material a mecanizar ${ }^{[5]}$.

Las aleaciones de titanio son materiales de uso estratégico en el sector aeroespacial debido a su densidad e interesantes propiedades mecánicas, que se mantienen incluso a temperaturas elevadas ${ }^{[6}$ y 7$]$.

Las aleaciones de titanio son materiales de difícil mecanizado ${ }^{[8]}$, debido a su baja conductividad térmica y a la elevada reactividad del titanio con los materiales de corte, recubrimientos y con el aire a alta temperatura ${ }^{[9}$ y 10]. Estas características provocan un elevado desgaste de herramienta, contaminación de la pieza de trabajo con material de corte, así como

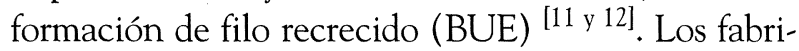
cantes de herramientas recomiendan para las aleaciones de titanio, parámetros de mecanizado moderados y abundante refrigeración, con el objetivo de controlar la temperatura en la zona de corte ${ }^{[13]}$.

La mayoría de los estudios acerca de mecanizado en seco de aleaciones de titanio se centran en procesos de torneado ${ }^{[14}$ y 15]. Asimismo, se recoge en la literatura el estudio del taladrado de un material híbrido formado por capas de material

(*) Dpto de Ingeniería Mecánica, Universidad Carlos III de Madrid, Avda. Universidad 30, 28911 Leganés (Madrid). Tel. +34 916245860. Fax. +34 916249430.e-mail: jcantero@ing.uc3m.es

(**) Dpto de Ingeniería Mecánica y Diseño Industrial. Universidad de Cádiz. Escuela Superior de Ingeniería. c/Chile s/n, E-11003, Cádiz

$\left(^{* *}\right)$ Dpto de Ingeniería de Construcción y Fabricación. UNED. Escuela Técnica Superior de Ingenieros Industriales. Apdo. 60249, E-28080, Madrid 
compuesto y aleación de titanio ${ }^{[16]}$. La mayoría de autores recomienda el uso de herramientas de metal duro, proporcionando valores de los parámetros de mecanizado del proceso correspondiente (velocidades de corte entre 45 y $100 \mathrm{~m} / \mathrm{min}$ y avances entre 0,25 y $0,35 \mathrm{~mm} / \mathrm{rev}$, tanto en torneado como en taladrado). La escasez de datos no permite establecer parámetros de corte óptimos, por lo que el mecanizado en seco de las aleaciones de titanio, es, todavía, un objetivo a conseguir.

En este artículo se ha estudiado el proceso de taladrado sin fluido de corte de la aleación Ti6A14V. Dado que la temperatura es un parámetro crítico que influye no sólo en el desgaste, sino también en el daño en el material mecanizado, se han comparado dos condiciones de proceso. En primer lugar, el taladrado continuo de series de taladros sin pausa entre ellos, enfriando después de cada serie la broca y el material con aire comprimido. En segundo lugar, el taladrado enfriando la herramienta y el material con aire comprimido tras cada taladro. El objetivo principal de este trabajo es analizar cómo influye en la vida de la herramienta y en la calidad del agujero obtenido, el diferente incremento de temperatura originado en una u otra condición.

\section{ENSAYOS DE TALADRADO}

Se realizaron ensayos de taladrado en un centro de mecanizado de control numérico KONDIA, modelo B500, con control numérico HEIDENHAIN TNC 415B.

Se mecanizaron planchas de la aleación Ti6Al4V de $8 \mathrm{~mm}$ de espesor, realizando taladros pasantes de $6 \mathrm{~mm}$ de diámetro, separados $15 \mathrm{~mm}$ entre centros.

Se seleccionó una broca de metal duro K40 (90\%WC, 10\%Co), con recubrimiento de TiN y punta afilada en cruz (DIN 6537). Se trata de una herramienta del fabricante GUHRING, adecuada para el mecanizado de aleaciones de titanio. Los parámetros de corte fijados para todos los ensayos fueron velocidad de corte de $50 \mathrm{~m} / \mathrm{min}$ y avance $0,07 \mathrm{~mm} / \mathrm{rev}$, que permiten realizar el estudio con una vida de herramienta razonable.

Las condiciones de proceso estudiadas corresponden a los siguientes tipos de ensayo:

\subsection{Ensayos de desgaste en taladrado continuo}

Se mecanizaron series de 8 taladros consecutivos (lo que permite llegar a una temperatura estacio- naria en la broca), sin pausa entre los mismos y, por tanto, sin enfriar la broca entre taladros.

Al final de cada serie, se enfriaron broca y material mediante un chorro de aire comprimido y se inspeccionó la broca visualmente y al microscopio óptico.

Para analizar los mecanismos de desgaste de la herramienta, se realizaron estudios SEM/EDS (Scanning Electron Microscopy/Energy Dispersive Spectrometer) después de la primera serie de taladros, tras 8 series y al fin de vida de la herramienta (16 series).

\subsection{Ensayos de desgaste enfriando material y broca después de cada taladro}

Se mecanizó con los mismos parámetros de corte, enfriando la broca y el material después de cada taladro. Se inspeccionó la broca visualmente y al microscopio óptico periódicamente. Asimismo, se estudió mediante SEM/EDS la herramienta.

\section{RESULTADOS Y DISCUSIÓN}

\subsection{Ensayos de desgaste en taladrado conti- nuo}

Se realizaron 16 series de 8 taladros (128 taladros) equivalentes a $7 \mathrm{~min}$ de tiempo de corte, tras los que se produjo la rotura de uno de los filos de la broca (fin de vida).

Tras la primera serie de 8 taladros se observó la herramienta al microscopio óptico y electrónico. En la figura 1 se muestra la imagen de electrones retrodispersados correspondiente. Para cada zona con diferente contraste del material se realizó un espectro semicuantitativo por EDS. La zona más oscura corresponde al recubrimiento de la herramienta (TiN), la zona gris son restos de la fase $\alpha$ de la aleación adherida a la herramienta y la zona blanca corresponde al sustrato de la broca (22\% Co-78\% W) con pérdida del recubrimiento.

El estudio con microscopía óptica confirmó la pérdida del recubrimiento de la herramienta, especialmente en la superficie de desprendimiento, y la adhesión de titanio concentrada en la punta de la herramienta y en la superficie de desprendimiento en la zona exterior de los filos (recrecimiento de filo). También, se observó la oxidación de la superficie de la broca en la zona cercana a la esquina de los filos (zonas de mayor velocidad de corte y por tanto mayor temperatura). Sin embargo, no se observó prácticamente ningún cambio geométrico de la herramienta. 


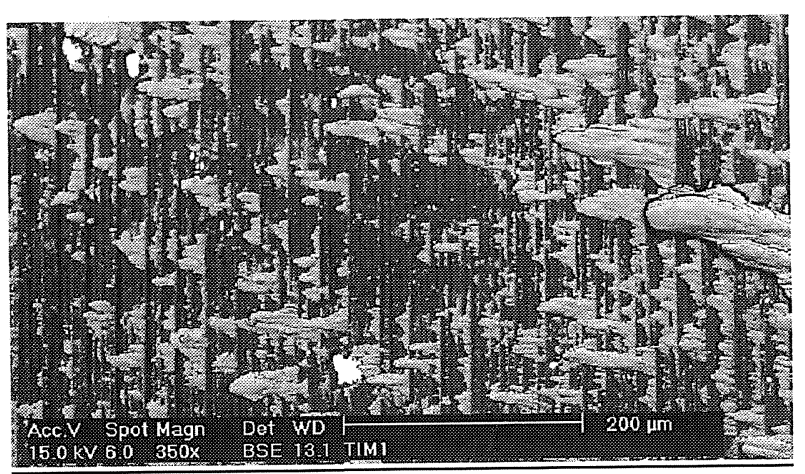

Figura 1. Micrografía de la broca después de mecanizar 1 serie de 8 taladros.

Figure 1. SEM micrograph view of drill affer machining 1 series of 8 holes.

El estudio de la broca después de las siguientes series de taladros mostró un desgaste más avanzado. En algunos taladros se apreciaron esporádicos fogonazos, correspondientes a pequeñas combustiones de la viruta de titanio, que fueron aumentando al realizar más taladros. Esto muestra un progresivo aumento de temperatura en la zona de corte. La figura 2 es una micrografía de la broca, realizada después del mecanizado de 8 series de 8 taladros continuos (mitad de la vida de herramienta). El análisis de la composición indicó que la zona más oscura corresponde a restos contaminantes con alto contenido en carbono debidos a la manipulación de la herramienta al realizar los ensayos; la zona gris clara es aleación adherida, la zona gris oscura corresponde al recubrimiento ( $\mathrm{TiN}$ ); y la zona blanca es material de corte que ha perdido el recubrimiento (esta zona se ha extendido considerablemente).

La observación mediante microscopio óptico a lo largo de la vida de la herramienta confirmó un

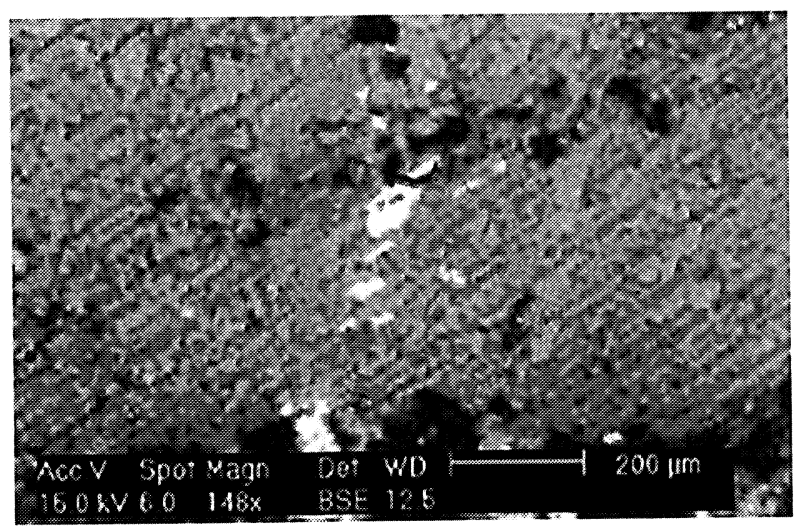

Figura 2. Micrografía de la broca después de mecanizar 8 series de 8 taladros.

Figure 2. SEM micrograph view of drill after machining 8 series of 8 holes.

Rev. Metal. Madrid Vol. Extr. (2005) 355-360 aumento progresivo de la pérdida de recubrimiento, adhesión de titanio y oxidación de la herramienta, mientras la geometría de la broca no sufrió cambios de geometría progresivos significativos (desgaste de flanco, redondeo de filo, etc.). Sin embargo, el deterioro de la herramienta es suficiente para aumentar la generación de calor, llegándose a la combustión de la viruta (Fig. 3) a partir del taladro 75 (tiempo de corte $4 \mathrm{~min}$ ). Este fenómeno perjudica tanto a la broca como al material. A partir del agujero 32 (tiempo de corte 1,7 min) se produjeron roturas en la broca, especialmente en las fajas de la herramienta (Fig. 4), que no requerían su sustitución.

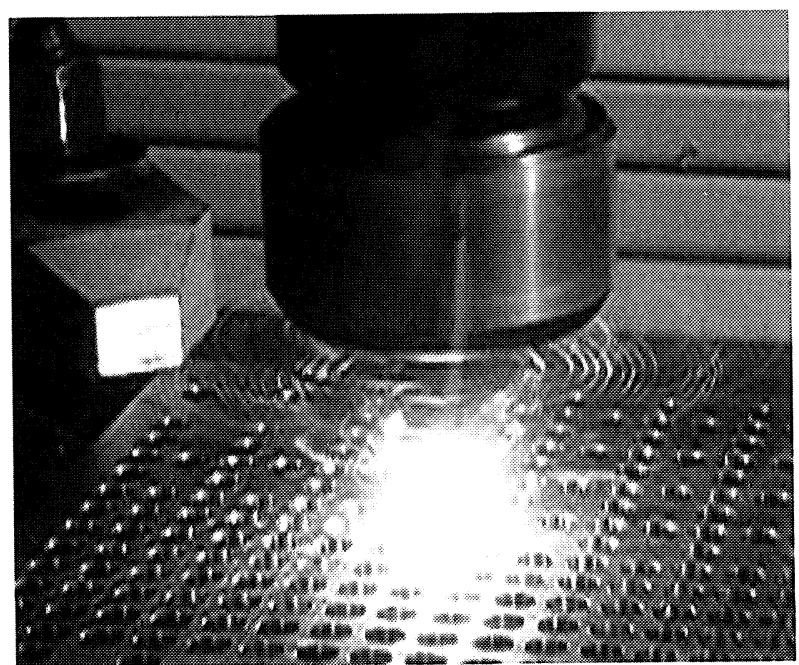

Figura 3. Proceso de taladrado con combustión de viruta.

Figure 3. Drilling test with combustion of the chip.

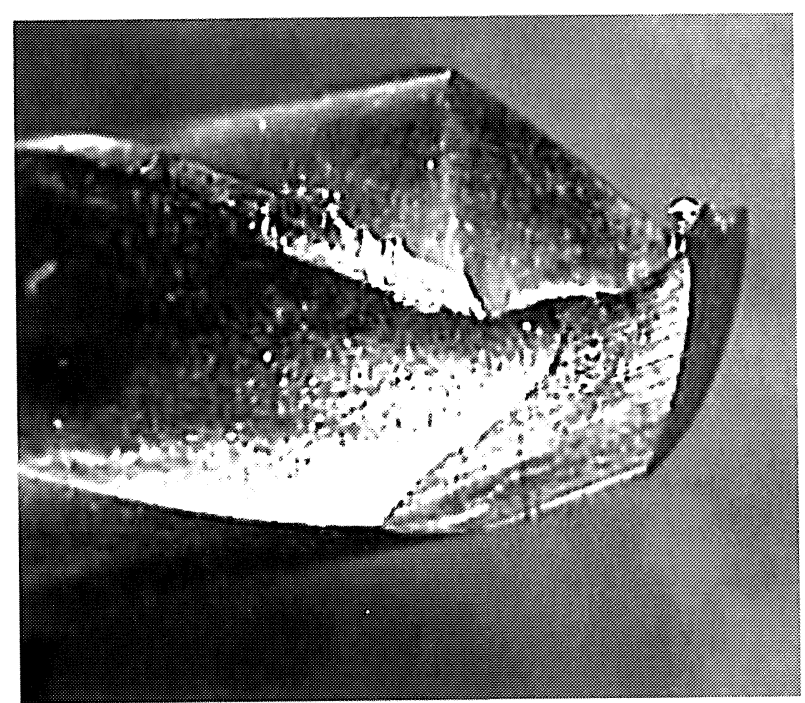

Figura 4. Broca después de mecanizar 6 series de 8 taladros continuos.

Figure 4. Drill after machining 6 series of 8 consecutively holes. 
El material circundante a los taladros parece intacto en algunos casos, pero presenta signos acusados de oxidación (Fig. 5) en los agujeros realizados con la broca más desgastada, coincidiendo con aquellos taladros en los que se produce combustión de la viruta. Este efecto es debido a las mayores temperaturas producidas.

El fin de vida de herramienta se alcanzó como consecuencia de la rotura de uno de sus filos (Fig. 6). Mediante el microscopio óptico se observó que incluso a fin de vida, la zona no afectada por la rotura sigue manteniendo su geometría inicial y presentando una buena agudeza del filo, similar a la de la herramienta nueva (Figs. 7 y 8 ).

Por último, las figuras 9 y 10 son micrografías de la superficie de desprendimiento de la herramienta al final de la vida de la misma. El análisis de composición indica que la zona más clara corresponde al material de corte (pérdida casi total del recubrimiento). La zona oscura es aleación adherida, que también se observó visualmente.

\subsection{Ensayos de desgaste enfriando material y broca después de cada taladro}

Se realizaron 455 taladros, equivalentes a $24 \mathrm{~min}$ de tiempo de corte (fin de vida).

Al igual que en los ensayos anteriores, se analizó la evolución del desgaste de la herramienta mediante SEM/EDS, inspección visual y microscopio

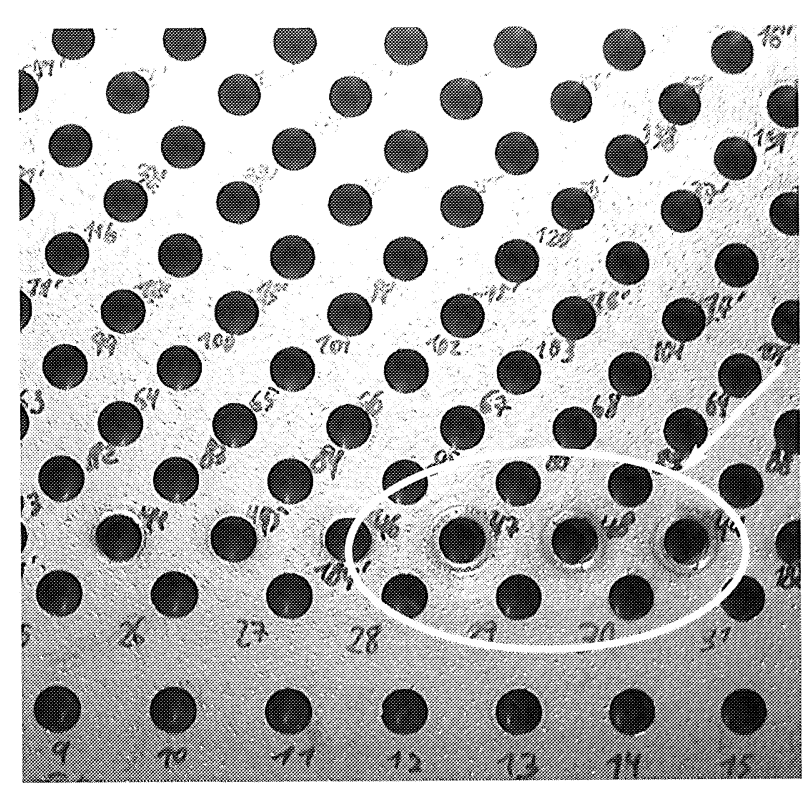

Figura 5. Fotografía de la placa mecanizada mostrando la entrada de los taladros.

Figure 5. Photograph of machined plate showing the drill entrance.

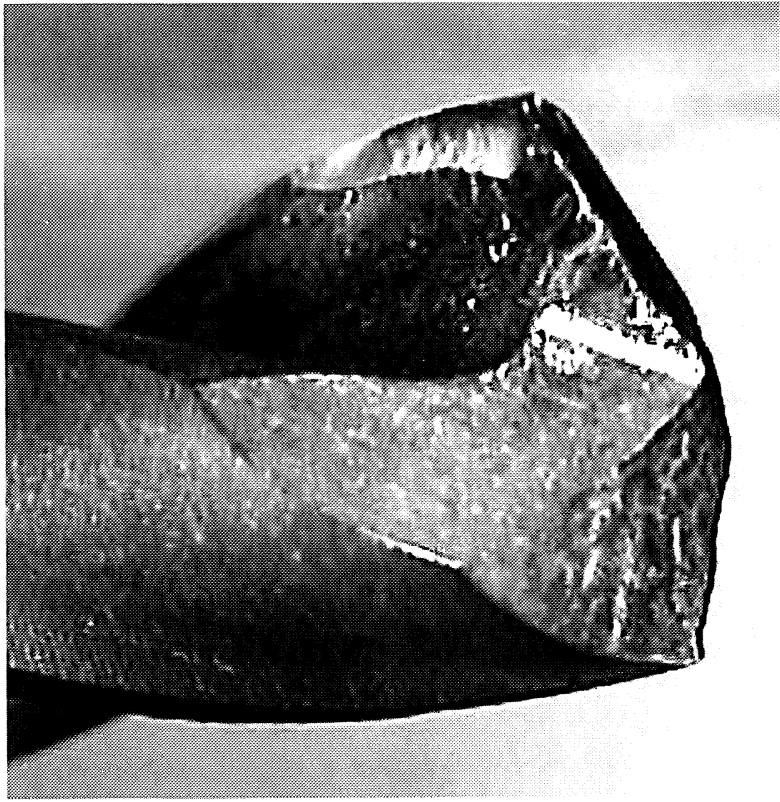

Figura 6. Broca al final de la vida (taladrado de 8 series sin enfriamiento).

Figure 6. Drill at the end of tool life (series of 8 holes without cooling).

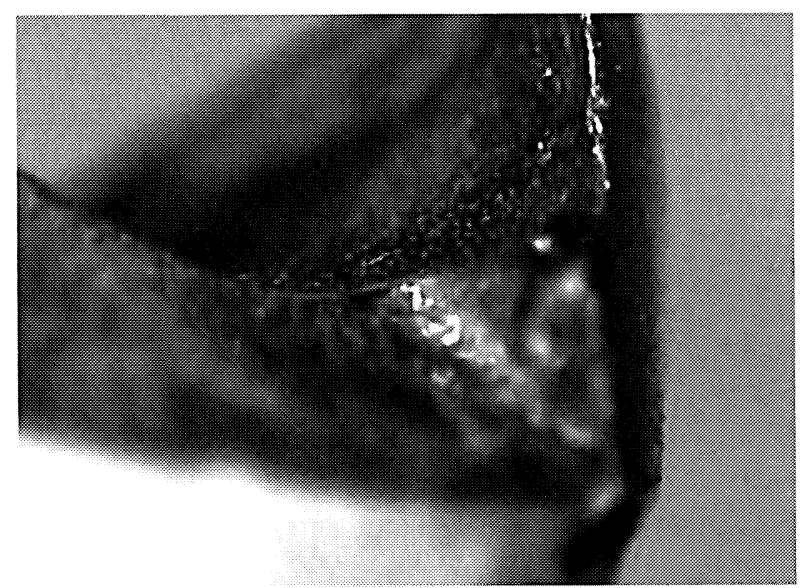

Figura 7. Filo de la broca a fin de vida (taladrado de 8 series sin enfriamiento).

Figure 7. Cutting edge drill at the end of tool life (series of 8 holes without cooling).

óptico. Los resultados obtenidos mostraron mecanismos de desgaste similares a los observados al realizar series de taladrado continuo, pero se produjeron con menor rapidez.

Los fenómenos observados para las condiciones anteriores, se repitieron en estos ensayos para un número de taladros mucho mayor (aproximadamente, entre tres y cuatro veces más taladros). Se produjeron roturas en la broca (fundamentalmente en la faja) a partir del agujero 92 (tiempo de corte $5 \mathrm{~min}$ ) que no supusieron su 


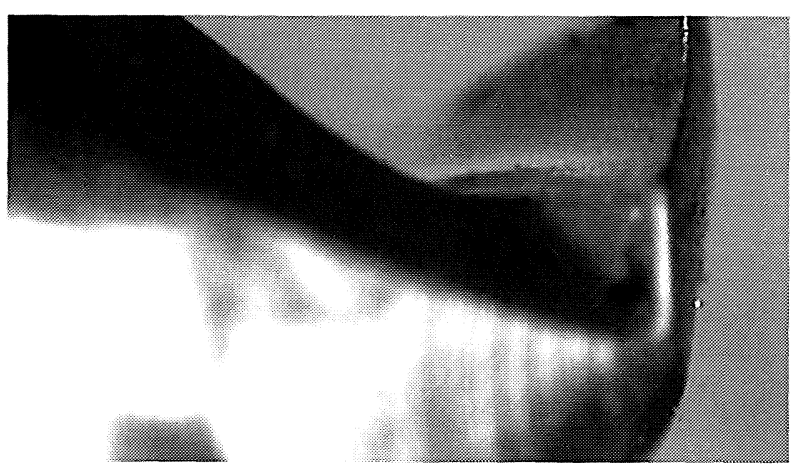

Figura 8. Filo de la broca nueva.

Figure 8. New drill cutting edge.

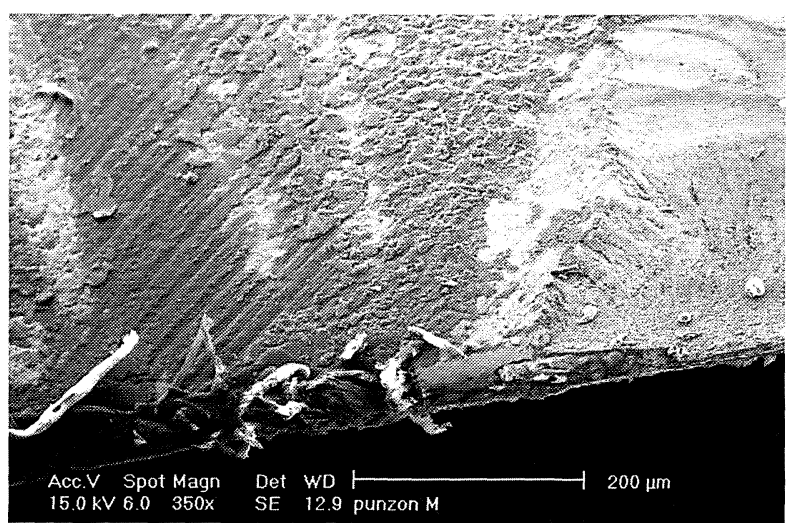

Figura 9. Micrografía de la broca a fin de vida (zona del filo).

Figure 9. SEM micrograph view of drill at the end of tool life (cutting edge).

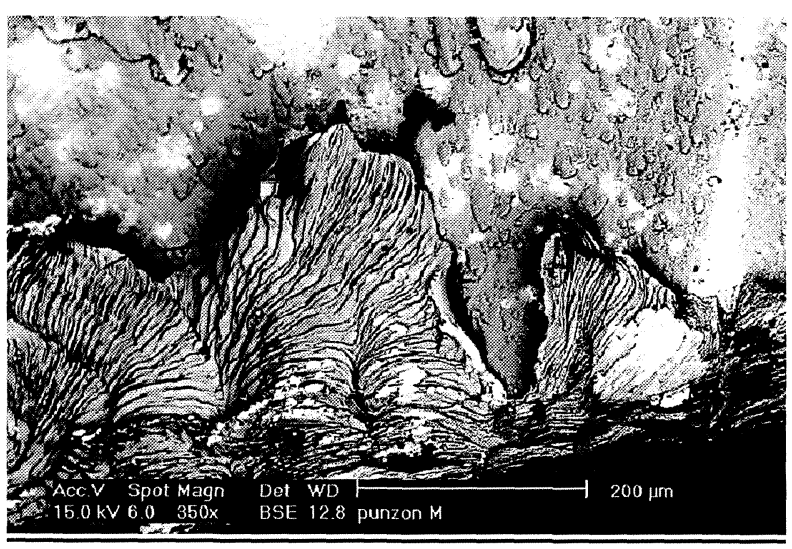

Figura 10. Micrografía de la broca a fin de vida (filo con gran cantidad de material adherido).

Figure 10. SEM micrograph view of drill at the end of tool life (material adhesion in the cutting edge).

sustitución. El aumento de temperatura en la zona de corte, debido al deterioro de la herramienta, provocó la combustión de la viruta en algunos agujeros a partir del 395 (tiempo de corte 21,2 min). Al igual que en los ensayos anteriores, el fin de vida de herramienta se alcanzó como consecuencia de la rotura de uno de sus filos, manteniendo en la zona no afectada por la rotura, la geometría y agudeza del filo inicial prácticamente intactas.

Con un micrómetro de interiores se ha medido el diámetro de los taladros de los dos tipos de ensayos. Los resultados son muy satisfactorios incluso con herramientas cercanas a su fin de vida, obteniéndose en todos los agujeros valores comprendidos entre $6,03 \mathrm{~mm}$ y $6,06 \mathrm{~mm}$. Aunque no se han hecho medidas de rugosidad en los agujeros, la inspección visual de los mismos indica un buen acabado en todos ellos. La rebaba en la salida y entrada de los agujeros también es poco significativa, excepto en los taladros en los que, debido a las elevadas temperaturas, se produce una combustión severa de la viruta.

\section{CONCLUSIONES}

El desgaste de la herramienta durante el taladrado en seco de la aleación Ti6Al4V no es progresivo, sino que el fin de vida se produce por rotura brusca de alguno de los filos.

Pequeñas modificaciones geométricas en la herramienta, la pérdida del recubrimiento y el deterioro superficial, provocan el aumento de temperatura en la zona de corte, llegando a producirse la combustión de la viruta. En los ensayos realizados se ha puesto de manifiesto que, con un desgaste de herramienta reducido es posible que el taladrado provoque un daño elevado al material circundante. En el caso del mecanizado de aleaciones de titanio, de uso en componentes de elevada responsabilidad, debería establecerse el criterio de fin de vida de herramienta, no en función de la medida de su desgaste, sino desde el punto de vista de la integridad del material y calidad del agujero obtenido.

El trabajo realizado pone de manifiesto la influencia de la temperatura en el desgaste de la herramienta y en el daño debido al mecanizado del material. El enfriamiento de la herramienta y del material entre taladros, redunda en una vida de herramienta más elevada junto con un mejor estado del material mecanizado.

Como líneas de trabajo futuras se pretende comprobar el estado microestructural del material, lo que permitiría establecer un criterio de fin de vida de la herramienta que garantice el control del daño, para unas condiciones de proceso establecidas. Este trabajo permitirá asimismo, determinar la 
conveniencia de ampliar el estudio del mecanizado en seco de aleaciones de titanio a otras condiciones de enfriamiento entre taladros o bien, la necesidad de abordar el mecanizado con refrigerantes ecológicos.

\section{Agradecimientos}

Este trabajo ha sido financiado por la Comisión Interministerial de Ciencia y Tecnología (CICYT) proyecto DPI2001-3747, por AIRBUS España, S.L. y por la Junta de Andalucía. Los autores agradecen al Departamento de Ciencia de los Materiales e Ingeniería Metalúrgica de la Universidad Carlos III de Madrid su ayuda y la disponibilidad de sus equipos para la realización de los estudios de microscopía óptica y SEM/EDS.

\section{REFERENCIAS}

[1] G. Byrne y E. Scholta., Ann. CIRP 42 (1993) 471-474.

[2] S. Hong, Y. Ding, W. JeOnG, Int. J. Mach. Tool Manu. 41 (2001) 2.271-2.285.

[3] Z.Y. WANG Y K.P. RAJURKAR, Wear 239 (2000) 168-175.

[4] P.S. SReejith y B.K.A. NgoI, J. Mater. Process. Tech. 101 (2000) 287-291.
[5] A. Jawaid, C.H. Che Haron y A. Abdullah J. Mater. Process. Tech. 92 (1999) 329-334.

[6] R.R. Boyer, Mat. Sci. Eng. A 213 (1996) 103-114.

[7] R.T. Holt, A.K. Koul, L. Zhao, W. Wallace, J.C. Beddoes y J.P. ImMARIGeON, Mater. Charact. 35 (1995) 41-67.

[8] E.O. Ezugwu, J. Bonney y Y. Yamane J. Mater. Process. Tech. 134 (2003) 233-253.

[9] L.N. López de LaCalle, J. PÉrez, J.I. Llorente y J.A SÁnCHEZ., J. Mater. Process. Tech. 100 (2000) 1-11.

[10] J.H. Xu y G.S. Geng J. Mater. Process. Tech. 129 (2002) 190-192.

[11] C. Ohkubo, I. Watanabe, J.P. Ford, H. Nakajima, T. Hosol y T. OKABE, Biomaterials 21 (2000) 421-428.

[12] S. LeI, W. LIU, Int. J. Mach. Tool Manu. 42 (2002) 653 661.

[13] SANDVIK COROMANT, Aerospace Frame. Application Guide (1991) 30-45.

[14] C.H. Che-Haron, J. Mater. Process. Tech. 118 (2001) 231-237.

[15] T. Kitagawa, A. Kubo y K. Maekawa, Wear 202 (1997) $142-148$.

[16] M. Ramulu, T. Branson y D. Kim, Compos. Struct. 54 (2001) 67-77. 